\title{
Applying Ant Colony Optimization Metaheuristic to the DAG Layering Problem
}

\author{
Radoslav Andreev*, Patrick Healy*, Nikola S. Nikolov* \\ radoslav.andreev@ul.ie, patrick.healy@ul.ie,nikola.nikolov@ul.ie \\ ${ }^{*}$ Department of Computer Science and Information Systems University of Limerick, IRELAND
}

\begin{abstract}
This paper ${ }^{1}$ presents the design and implementation of an Ant Colony Optimization based algorithm for solving the DAG Layering Problem. This algorithm produces compact layerings by minimising their width and height. Importantly it takes into account the contribution of dummy vertices to the width of the resulting layering.
\end{abstract}

\section{INTRODUCTION}

The Sugiyama framework [12] is the most well-known and studied heuristic for drawing directed acyclic graph (DAG). It comprises a number of steps one of which is assigning each vertex of the graph to a layer (layering step), which causes a dummy vertex to be created every time an edge crosses a layer. The next step is to order the vertices inside each layer so that the number of edge crossings between any two layers is minimised. The layering step is the one that determines what will be the height and the width of the final drawing. Usually the height represents the number of layers used to layer the graph and the width is the maximum count of real vertices in a layer. Defined like this the width ignores the contribution made by the dummy vertices. In the case where the width of a real vertex is much greater than the one of a dummy vertex, or the number of dummy vertices for any given layer is much smaller than the number of real vertices this definition is accurate enough. However, when dummy vertices are not so small compared to the real ones or when their number is high, ignoring them will inevitably result in much wider final drawing than it was expected initially. In this paper we present a layering method based on the Ant Colony Optimisation (ACO) metaheuristic [4] that minimises the width and height of the layering by taking into account the contribution of dummy vertices. To the best of our knowledge this is the first attempt to use the ACO based algorithm to layer a DAG.

In the following we introduce some preliminaries and discuss existing layering methods, followed by an introduction to the ACO metaheuristic and the representation of the layer assignment problem in its terms. Further, we describe the design and the implementation of our algorithm together with a discussion about the results achieved. Finally, a conclusion and directions for further research are given.

\section{PRELIMINARIES}

A layering of $G$ is a partition of $V$ into subsets $L_{1}, L_{2}, \ldots, L_{h}$, such that if $(u, v) \in E$, where $u \in L_{i}$ and

\footnotetext{
${ }^{1}$ Supported by SFI Basic Research Grant 04/BR/CS0696
}

$v \in L_{j}$, then $i>j$. The span of an edge $(u, v)$ with $u \in L_{i}$ and $v \in L_{j}$ is $i-j$. A layering is proper if all edge spans equal one. This is achieved by inserting dummy vertices along edges whose edge span is greater than one. The layer span $L(v)$ of vertex $v$ refers to the set of layers between the topmost and the lowermost layer on which vertex $v$ can be placed, provided that all edges point downwards. Note that the layer span for a vertex is not constant during the layering process and changes depending on the layer assignments of its neighbouring vertices.

The most common definition (without any restrictions on the layering properties) of the DAG Layering Problem (LP) can be stated as: Given a DAG, $G=(V, E)$, find a valid layer assignment so that for each vertex $u$ with $y$-coordinate $y(u)$ and for each vertex $v$ such that $(u, v) \in E$ the following properties are satisfied [1]:

1) $y(u)$ is an integer

2) $y(u) \geq 1$

3) $y(u)-y(v) \geq 1$

There are three important aspects of the DAG LP according to [1]:

1) The layered DAG should be compact. This means that its vertices should be evenly distributed over the drawing area.

2) The layering should be proper. This is easily achieved by inserting dummy vertices.

3) The number of dummy vertices should be small.

A layering algorithm trying to solve the DAG LP subject to additional constraints is expected to produce a layering with specified width or height or minimum number of dummy vertices or a combination of those three layering properties. The height of a layering is defined as the number of layers used to layer the DAG. Normally the vertices of DAGs from reallife applications have text labels and sometimes prespecified shape. Here we use a definition of vertex width given in [9] and stating that the width of a vertex is the width of the rectangle enclosing the vertex. If the vertex has no text label and no information about its shape or size is available we assume that its width is one unit. The width of a layer is defined as the sum of the widths of all vertices in that layer (including the dummy vertices) and the width of a layering is the maximum width of a layer [9].

The edge density between horizontal levels $i$ and $j$ with $i<j$ is defined as the number of edges $(u, v)$ with $u \in$ 
$L_{j} \cup L_{j+1} \cup \ldots \cup L_{h}$ and $v \in L_{1} \cup L_{2} \cup \ldots \cup L_{i}$. The edge density of a layered DAG is the maximum edge density between adjacent layers (horizontal levels) [9]. Naturally, drawings with low edge density are more readable and easier to comprehend.

A definition of the DAG LP with additional constraints governing the width and height of the resulting layering subject to minimum number of dummy vertices is given in [11].

\section{EXISTING LAYERING METHODS}

One of the most well known layering algorithms is the Longest-Path Layering (LPL) described in Algorithm 1. In the representation of this and the following algorithms we denote the set of all immediate predecessors of vertex $v$ by $N_{G}^{-}(v)$, and the set of all immediate successors of vertex $v$ by $N_{G}^{+}(v)$. The LPL method places all sink vertices in layer $L_{1}$, and each remaining vertex $v$ is placed in layer $L_{p+1}$, where $p$ is the longest (maximum number of edges) path from $v$ to a sink. The attractiveness of this method is that it has linear time complexity (because the graph is acyclic) and it uses the minimum number of layers possible. The disadvantage of the LPL method is that its layerings tend to be too wide [6]. Because the area occupied by the final drawing depends on both its width and its height the Longest-Path Layering is not the best choice if minimal layering area is the main priority. Unfortunately, the problem of finding a layering with minimum width, subject to having minimum height, is $N P$ complete [1].

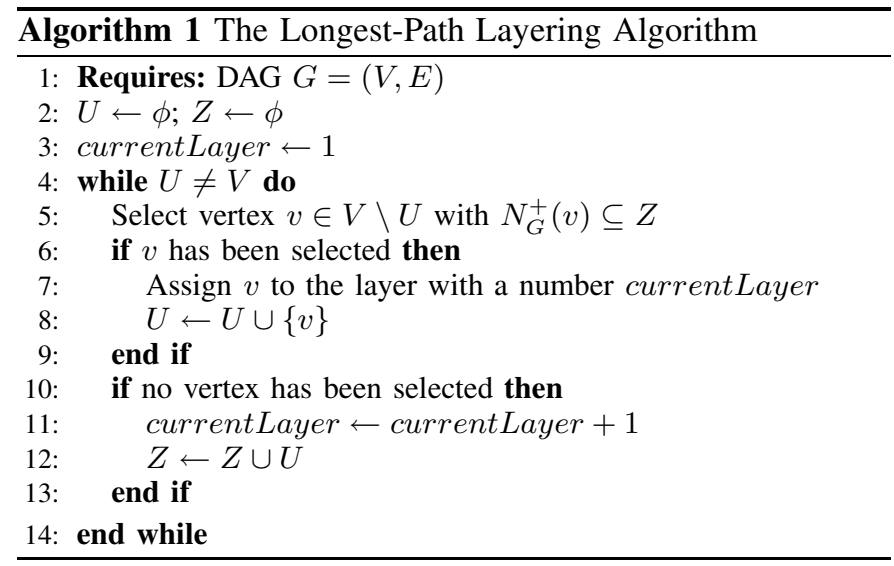

Another layering method is the MinWidth heuristic [9] displayed in Algorithm 2. It is roughly based on the LPL algorithm. The authors employ two variables widthCurrent and widthUp to keep the width of the current layer, and the width above it, respectively. The width of the current layer, widthCurrent, is calculated as the number of original vertices already placed in that layer plus the number of potential dummy vertices along edges with a source in $V \backslash U$ and a target in $Z$ (one dummy vertex per edge). The variable width $U p$ is an estimation of the width of any layer above the current one. It is the number of potential dummy vertices along edges with a source in $V \backslash U$ and a target in the current layer (one dummy vertex per edge). When a vertex is selected to be placed an additional condition conditionselect is used, which is true if $v$ is the vertex with the maximum out-degree among the candidates to be placed in the current layer. Such a choice of $v$ results in maximum reduction to widthCurrent. For a thorough discussion of the MinWidth heuristic the reader is referred to [9].

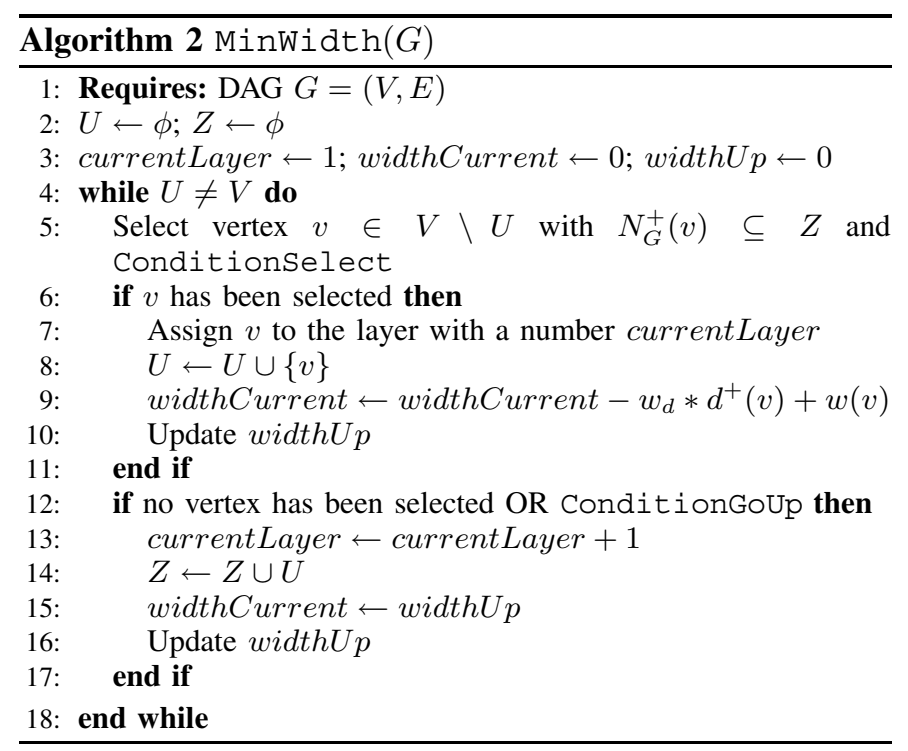

Promote Layering (PL) [8] is a heuristic whose goal is "to develop a simple and easy to implement layering method for decreasing the number of dummy vertices in a DAG layered by some list scheduling algorithm." The PL layering method is an alternative to the network simplex method of Gansner et. al [5] but considerably easier to implement and especially useful when a commercial linear programming solver is not available. As noted PL usually runs after a layering is produced by a quick list scheduling algorithm like LPL. LPL and MinWidth on their own and in combination with the PL heuristic were the four benchmark algorithms used in this work to evaluate the performance of our ACO-based layering algorithm.

\section{INTRODUCTION TO THE ACO METAHEURISTIC}

Ant colonies, and more generally social insect societies, are distributed systems that, in spite of the simplicity of their individuals, represent a highly structured social organisation. As a result of this organisation, ant colonies can accomplish complex tasks that in some cases far exceed the individual capabilities of a single ant [4].

The main idea behind the Ant Colony Optimisation (ACO) metaheuristic is that self-organising principles, which allow the highly coordinated behaviour of real ants, can be exploited to coordinate populations of artificial agents that collaborate to solve computational problems.

The real ants coordinate their activities via stigmergy. This is a biological term about a form of indirect communication mediated by modifications of the environment. The term was first introduced by the French biologist Pierre-Paul Grasse in 
1959 to refer to termite behaviour. He defined it as "Stimulation of workers by the performance they have achieved". An ant coming back to its nest from a food source it has found will deposit a chemical substance called pheromone which the others will find while roaming for food. By following the pheromone trail laid the rest of the ants will discover that same food source. The idea is then to use a similar artificial stigmergy, as a form of global knowledge, to coordinate societies of computational agents in an attempt to solve different combinatorial optimisation problems.

Such a computational agent is defined as "a stochastic constructive procedure that incrementally builds a solution by adding opportunely defined solution components to a partial solution under construction" [4]. Based on the above definition an ACO metaheuristic can be applied to any combinatorial optimisation problem for which a constructive heuristic can be defined. "The real issue is to find a suitable problem representation which the artificial ants will use to build their solutions" [4].

\section{A. ACO definitions}

A tour is a single iteration during which every ant produces a solution to the problem being solved. For a given tour all ants use the same starting point reached by the previous tour. This approach emulates a parallel work environment for all the ants comprising the colony. At the end of a tour, depending on the pheromone update strategy adopted, either one or more ants with the highest objective function value will deposit certain amounts of pheromone over the edges of the construction graph comprising its/their solution(s).

The process of constructing a solution by a single ant is called a walk [4]. In our algorithm each ant is placed on a randomly selected vertex $v$ from which it starts constructing its layering. Once a vertex is assigned the next one is chosen by the ant again randomly and is assigned to a layer. This continues until each vertex is assigned to a layer.

When performing its walk an ant executes a finite number of identical actions called a construction step [4]. At each construction step an ant $k$ applies a probabilistic action choice rule, called random proportional rule [4] given by Eq.(1), to decide which layer vertex $v$ should be assigned to.

\section{B. DAG LP representation in terms of the ACO metaheuristic}

The first step when applying ACO to a combinatorial optimisation problem is to define the construction graph $G_{C}$ on which ants will perform their walks. The DAG LP can be cast into the framework of the ACO metaheuristic using the construction graph $G_{C}=(C, H)$. Here $C=V \bigcup L$ is the set of components which includes $V$, the vertex set of graph $G$ that is to be layered, and $L$, the set of layers. $H$ is the set of links connecting components (vertices and layers) from $C$. Note that only a lower bound of $|L|$ is known beforehand but not $|L|$ itself. Each layer assignment, which consists of $n$ couplings $\left(v_{i}, l_{j}\right)$ of vertices and layers, corresponds to at least one ant's walk on the construction graph and cost $d_{i j}$ is associated with every possible coupling of vertex and layer. In the original definition of the construction graph $G_{C}, H$ fully connects the components of $C$ [4]. This is not the case here because when assigning vertex an ant will be limited to choose from layers comprising the layer span of that particular vertex.

\section{Constraints}

Walks on the construction graph $G_{C}$ have to satisfy the width and height constraints for the layering in order to result in a valid assignment. One particular way of generating such an assignment is by an ant's walk that randomly chooses vertex $v \in G$ as a starting point and continuing with a random selection of next vertex until all vertices of $G$ are assigned. Additionally layers' resource capacities (width $W$ ) can be enforced by an appropriately defined neighbourhood. For example, for an ant $k$ positioned on vertex $v_{i}$ the neighbourhood $N_{i}^{k}$ can be defined as consisting of the subset of layers of $L\left(v_{i}\right)$ to which $v_{i}$ can be assigned without exceeding $W$.

\section{Pheromone trails and heuristic information}

Ants construct feasible solutions by iteratively adding new components to their partial solutions. While in the construction process ants repeatedly have to take the following two basic decisions:

1) choose the vertex to assign next;

2) choose the layer the vertex should be assigned to.

Pheromone trail information can be associated with any of the two decisions. It can be used to learn an appropriate order for vertex assignment or it can be associated with the desirability of assigning vertex $v_{i}$ to a specific layer. In the former case, $\tau_{i j}$ represents the desirability of assigning vertex $v_{i}$ immediately after vertex $v_{j}$, while in the latter it represents the desirability of assigning vertex $v_{i}$ to layer $l_{j}$. In our implementation we use pheromone trail information to measure the desirability of assigning a given vertex to any of the layers from its layer span, that is, the second case. Similarly, heuristic information $\eta_{i j}$ can be associated with any of the two decisions listed above but again we use heuristic information for the actual assignment and not for the order in which it is going to be done. Methods such as Breadth First Search or other similar techniques which provide a linear order of the vertices can be used to determine the order in which vertices are to be assigned. Random choice of the next vertex to be assigned is another option that may be employed.

The heuristic information can be either static or dynamic. In the static case, the values $\eta_{i j}$ are computed once at the initialisation phase of the algorithm and then remain unchanged throughout the entire algorithm's run. In the dynamic case the heuristic information depends on the partial solution constructed so far and therefore has to be computed at each step of an ant's walk. Our application of the ACO to the DAG LP falls into the second category because the heuristic value $\eta_{i j}=\frac{1}{w_{i j}}$ where $w_{i j}$ is the width of a layer $l_{j} \in L\left(v_{i}\right)$. Therefore after each assignment, which in fact moves $v_{i}$ from its current layer $l_{\text {curr }}$ to a new one $l_{\text {new }}$, the widths of those two layers must be changed - decreased for $l_{\text {curr }}$ and increased for $l_{\text {new }}$. Moreover, the widths of the layers from 
$L\left(v_{i}\right)$ placed between $l_{\text {curr }}$ and $l_{\text {new }}$ also change because of the dummy vertices induced by incoming and outgoing edges for vertex $v_{i}$. Therefore the heuristic values affected must be computed by every ant after each assignment it has made. When constructing its walk on the construction graph an ant $k$, that is going to assign vertex $v_{i}$, chooses layer $l_{j} \in L\left(v_{i}\right)$ with a probability given by the following equation [4]:

$$
p_{i j}^{k}=\frac{\left[\tau_{i j}\right]^{\alpha}\left[\eta_{i j}\right]^{\beta}}{\Sigma_{l \in N_{i}^{k}}\left[\tau_{i l}\right]^{\alpha}\left[\eta_{i l}\right]^{\beta}}
$$

Here $\eta_{i j}$ is the heuristic information that is calculated a priori and $\tau_{i j}$ is the quantity of pheromone calculated as the product of the initial pheromone value, the evaporation process and the quantity deposited by ants in previous tours. The two parameters $\alpha$ and $\beta$ determine the relative influence of the pheromone trail and the heuristic information. $N_{i}^{k}$ is the feasible neighbourhood of ant $k$ when assigning vertex $v$, that is the layer span of $v$. The role of $\alpha$ and $\beta$ is the following. If $\alpha=0$, the layers from the layer span of $v$ with smaller widths are more likely to be selected because the influence of the pheromone information is eliminated. This corresponds to a classic stochastic greedy algorithm with multiple starting points since ants are initially randomly distributed over the vertices of the graph to be layered [4]. Conversely if $\beta=0$, only the pheromone information is at work, and therefore the layers that had been selected by the majority of ants during previous tours that is, have accumulated high pheromone values, will more likely be selected. The absence of heuristic bias generally leads to rather poor results, and in particular, for values of $\alpha>1$ it leads to rapid emergence of a stagnation situation where all the ants follow the same tour, which in general is strongly suboptimal [3].

\section{E. Representing ants}

There are a few key features that ants need to have in order to be able to perform their walks on the construction graph and generate feasible solutions. An ant has to be able to:

1) Memorise the partial solution it has constructed so far;

2) Determine the feasible neighbourhood for each vertex;

3) Assign a vertex to a layer subject to the height and width constraints;

4) Update the values of the heuristic matrix to reflect each new assignment;

5) Update the layer span for a vertex;

6) Compute and store the objective function value of the solution it generates;

7) Update the pheromone matrix.

The first requirement can be satisfied by storing the partial solution (walk) into an array indexed by the vertices of $G$ and associating an integer value with each vertex representing the layer number it has been assigned to. An ant should also be able to compute the layer span of a given vertex in order to determine its neighbourhood. Additional to the layer span an ant should be able to calculate the number of dummy vertices a particular assignment would cause due to incoming edges for vertex $v$ which cross the layers above the one to which $v$ is assigned. These must be performed after each assignment. Finally, each ant should have a number of variables in which the characteristics of the completed layering will be kept; these are the value of the objective function, the height of the layering and the width of the layering.

\section{THE ACO-BASED LAYERING ALGORITHM}

In our approach the graph is first layered using the fast and efficient LPL algorithm which gives the minimum number of layers graph $G$ can be layered on. We then add a number of new layers in between the LPL ones. This results in a greater search space for the ants and gives them more freedom when constructing their solutions. Once the LPL layering is stretched in this manner the actual process of layering by the ant colony begins. It comprises a number of tours during which each ant builds its own layering. The layering of the best ant becomes this tour's layering. Every tour inherits the layering of its predecessor and uses it as a base to build its own. The rest of this section presents the details of our ACO-based layering algorithm.

\section{A. Stretch $L P L$}

The aim of this initial step of the algorithm is to add new layers to the ones introduced from LPL so that the number of layers grows to $n$, the number of vertices of $G$. By doing this we guarantee that no layering will be left out, that is these with the minimum width will also be in the search space. This approach also enlarges the search space, giving ants greater area for exploration. This would not be the case if they start working directly on the resulting layering from the LPL. This layering is a minimum height layering and as such it is too restrictive for ants. The only improvement they could make is to reduce the number of dummy vertices similar to the PL heuristic described in Section III. However, ants will not be able to reduce the width of the graph significantly. If we denote the number of layers produced by LPL as $n_{L P L}$ and with $n$, the number of vertices of $G$, then the number of layers to add is given by $n_{n l}=n-n_{L P L}$.

One way to proceed is to add all new layers either above or below the LPL layers. Alternatively some of them can go above and the other below the LPL layers (Fig. 1).

The drawback of the both strategies is that the ant will have very few options as to where to move the vertex. Bearing in mind that each ant chooses a starting vertex randomly, it will be very limited in moving vertices without violating the initial direction of the edges of $G$. Of course, if the vertex is either a sink or a source an ant will have at its disposal (at least) half of all newly added layers but then it is hard to determine on which layer exactly to place the vertex as no heuristic information would be available to bias the ant's choice.

The approach suggested here is to insert the new layers in between the LPL layers. The way to do it is to divide the $n_{n l}$ to the number of interlayer spaces from the LPL which is exactly $n_{L P L}-1$ and then insert and re-index the layers as shown in Fig. 2. 


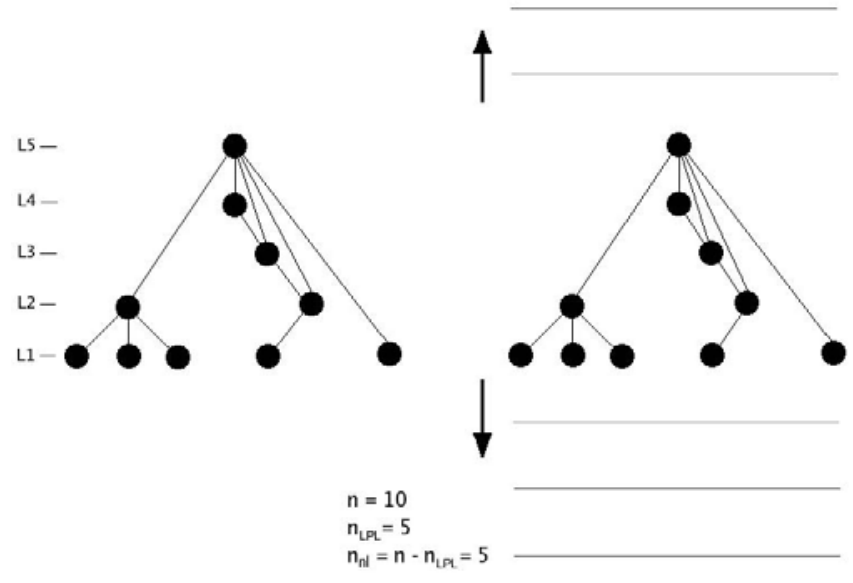

Fig. 1. The LPL and the newly added layers on top and bottom.
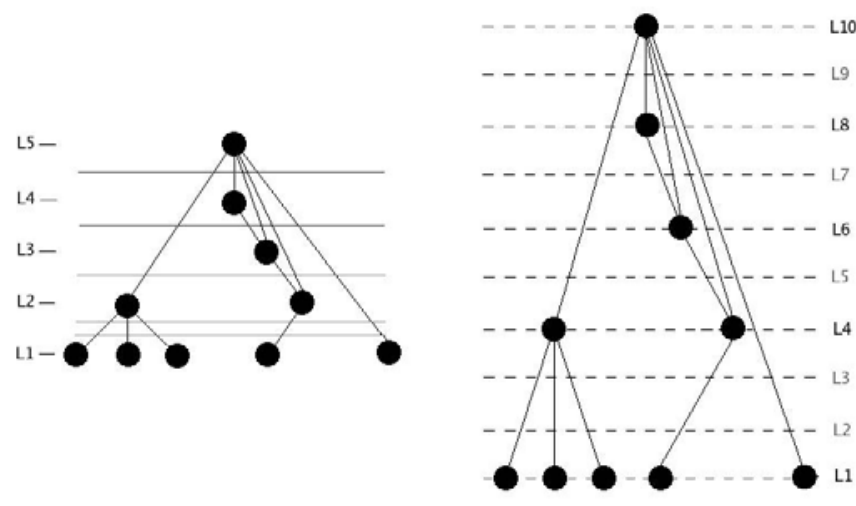

Fig. 2. The new layers inserted between the LPL ones.

By choosing this approach over the one described in Fig. 1 the layer span for each vertex is uniformly increased and therefore ants will have more possibilities for changing the layer assignment of any vertex and not only the source or sink ones. We denote the resulting (stretched) graph as $G_{S T R}$.

Vertex width is another issue that needs careful consideration. In most real-life applications the width of dummy vertices (which in fact would be the line representing an edge) is far less than the width of a real vertex (usually a rectangle with some text inside). To reflect this the ACO-based layering algorithm allows for a variable dummy vertex width to be supplied as an initial parameter.

\section{B. Initialisation phase}

In the initial phase the input DAG $G=(V, E)$ is layered by the LPL. The resulting layering is then stretched as described in Section V-A allowing for a much greater exploration area for the ants. The next step is to calculate the layer span $L\left(v_{i}\right)$ $\forall v \in V$. Based on the layer span of a particular vertex, its corresponding elements from the heuristic matrix (one column per vertex) are initialised either to 0 or $\frac{1}{W\left(l_{i}\right)}$ depending on whether $l_{i}$ belongs to the layer span of that vertex or not. Here $W\left(l_{i}\right)$ is the width of layer $l_{i}$. However all elements of the pheromone matrix are initialised to $\tau_{0}$, the initial amount of pheromone laid down.

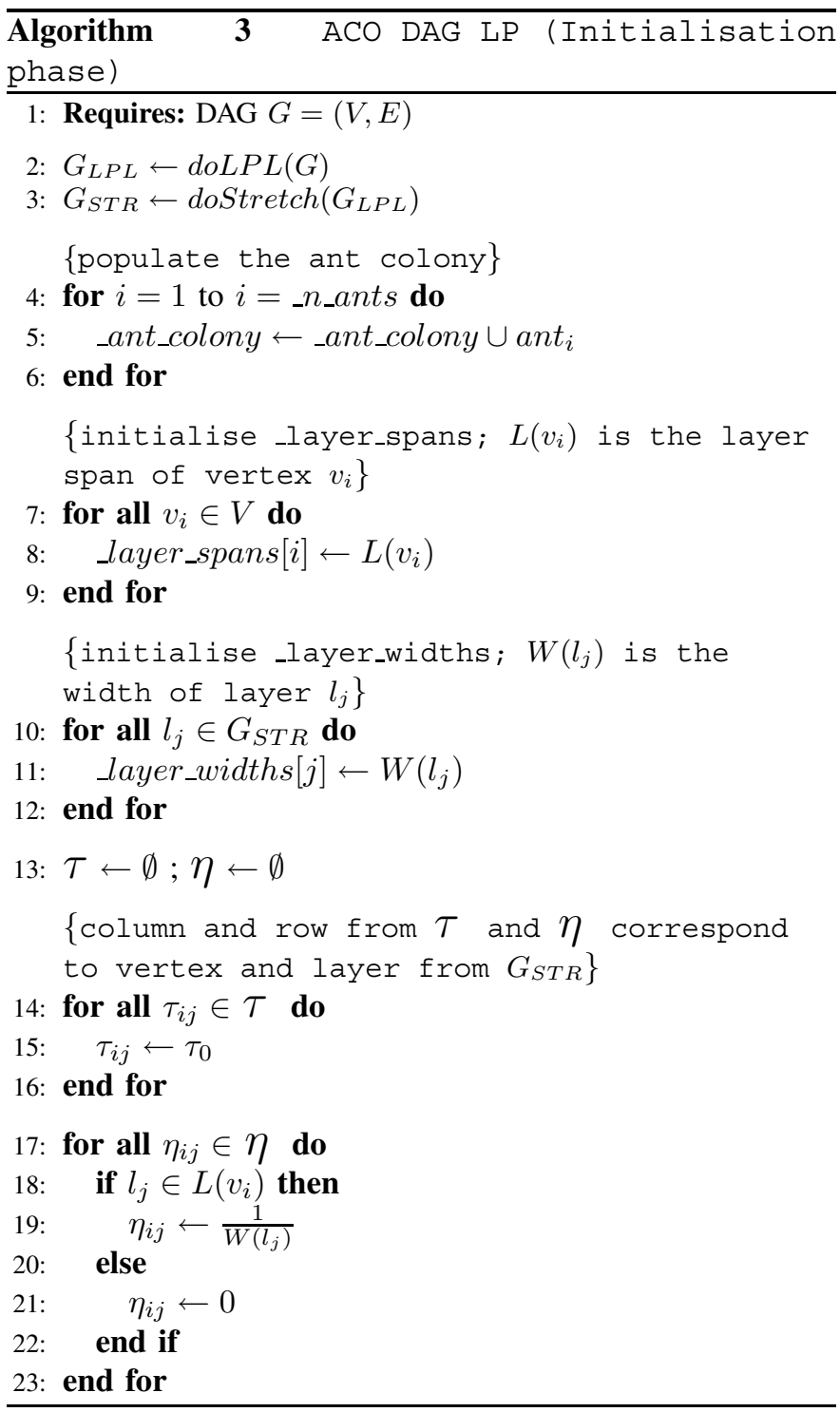

\section{Layering phase}

Once the initialisation phase is completed Algorithm 4 Layering Phase starts. Its outermost loop runs for the specified number of tours _n_tours; 10 was the value we used in our experiments. During a single tour each ant performs its walk on the construction graph $G_{C}$ and produces a layering of $G_{S T R}$. When building its solution ant $a_{k}$ repeatedly assigns vertex $v_{i}$ (randomly chosen) to a layer $l_{\text {best }} \in L\left(v_{i}\right)$ that gives best result when executing line 6 .

At line 7 the actual assignment is performed, which in turn requires that those values of the heuristic matrix $\eta$ that have been affected by this particular assignment be recalculated and updated (line 8). When $v_{i}$ is assigned to a layer, that is, it has been moved either up or down from its current layer, the layer 
span of all neighbouring vertices of $v_{i}$ changes too. Therefore the layer span for every neighbouring vertex of $v_{i}$ has to be recalculated (line 10) before ant $a_{k}$ picks up the next one. When $a_{k}$ has assigned all vertices it is the end of its walk for the current tour. The objective function value is calculated at line 13 and stored against that ant's identifier.

At the end of a tour the evaporation step, which reduces all elements $\tau_{i j}$ of the pheromone matrix $\tau$ by a predefined evaporation rate $\rho_{0}$, is executed. Next, the best ant for the tour $a_{\text {best }}$ deposits pheromone on the elements $\tau_{i j}$ corresponding to its assignments. Additionally the heuristic matrix and layering of $a_{\text {best }}$ become the starting heuristic matrix and layering for the next tour.

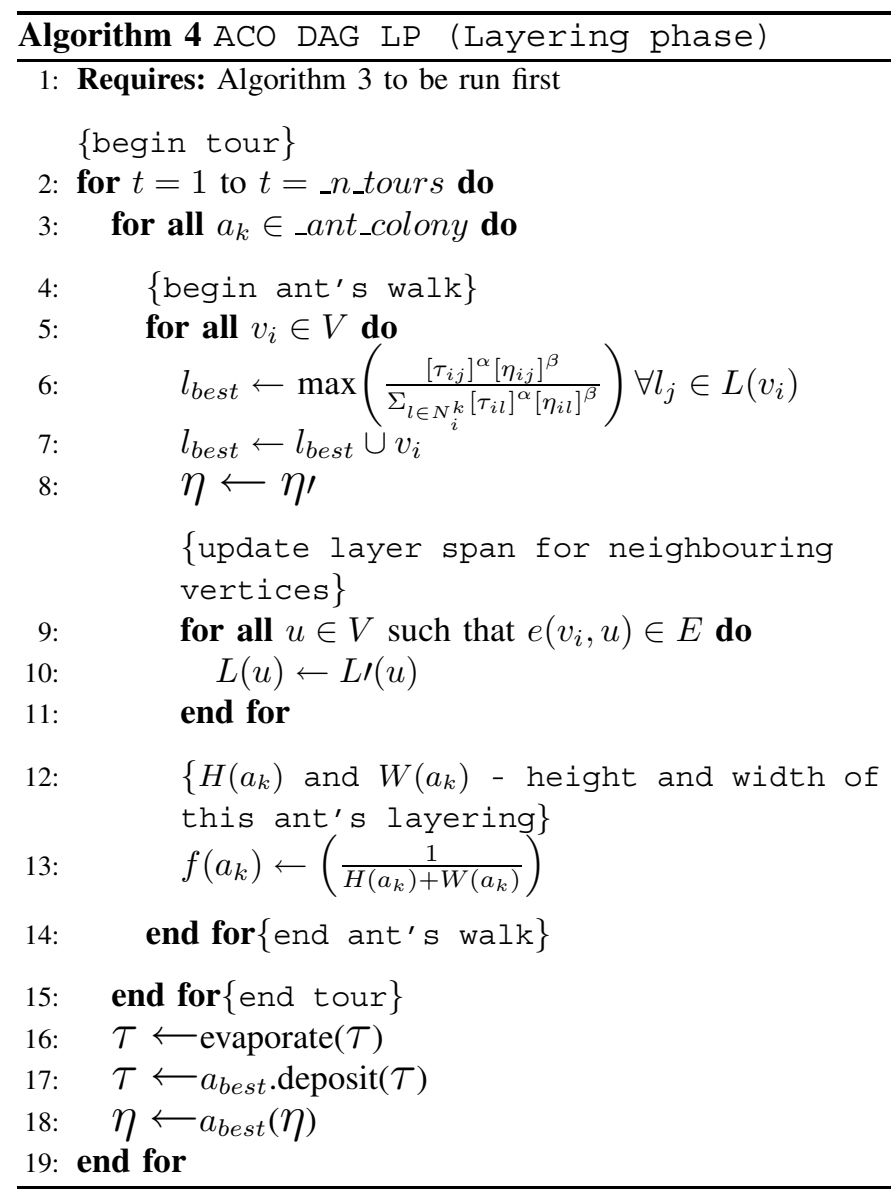

\section{IMPLEMENTATION OF THE ACO-BASED LAYERING ALGORITHM}

The algorithm was implemented in $\mathrm{C}++$ with the use of the LEDA 5.0 library of efficient data types and algorithms ${ }^{1}$. Three classes were used, LayeredDAG, Ant, and Antcolony. The class LayeredDAG inherits from LEDA's parameterised graph GRAPH<int, int > and has additional methods to allow for layering of the graph. The class Ant represents a single computational agent, which performs walks on the construction graph $G_{C}=(C, H)$,

\footnotetext{
${ }^{1}$ http://www.algorithmic-solutions.com/enleda.htm.
}

while building its own solution in parallel with other agents (ants). Finally, the AntColony class is the entity conducting the search process performed by ants.

The most important method of the Ant class is called performWalk ( ) . First it initialises this ant's pheromone and heuristic matrices, its objective function value, as well as its own copy of the layer widths data structure. Then it iterates randomly over all vertices of the graph to be layered. After a vertex is picked up, the calcProbability () method is called, which calculates probability values for each layer from the vertex's layer span according to the random proportional rule given by (1). The layer that corresponds to the highest probability value is chosen and the vertex is assigned to it. To accomplish this operation the algorithm invokes two other methods - moveNode () and reflectNodeMovement ().

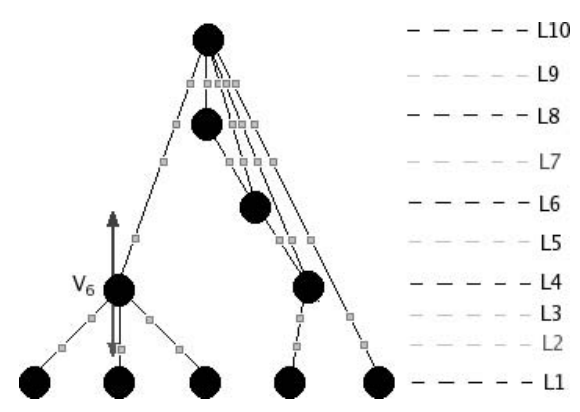

Fig. 3. Reflect vertex movement

\section{Updating layer widths}

When a vertex is moved by an ant, the widths of all layers between and including the current layer and the new one, have to be updated. The algorithm used to accomplish this is Algorithm 5. Here _n_width is the width of a real vertex and nnd_width is the width of a dummy vertex. Additionally, outdeg $\left(v_{i}\right)$ and indeg $\left(v_{i}\right)$ are the numbers of outgoing and incoming edges for $v_{i}$ respectively. Please refer to Fig. 3 when reading the algorithm.

The main method of the Antcolony class is runcolony(). It calls the performTour() method for the specified number of tours (i_maxiterations), and performTour() calls performWalk() method on each ant from the colony. That method returns the objective function value the ant has achieved.

Note: When the ants produce their layering it might happen that some of the layers between the first and the last layer remain empty. To eliminate this after the layering is completed empty layers in the middle are removed and the layer numbers assigned to vertices are updated.

\section{EXPERIMENTS AND RESULTS}

Experiments to evaluate the performance of Ant Colony layering algorithm were conducted over a set of 1277 directed 


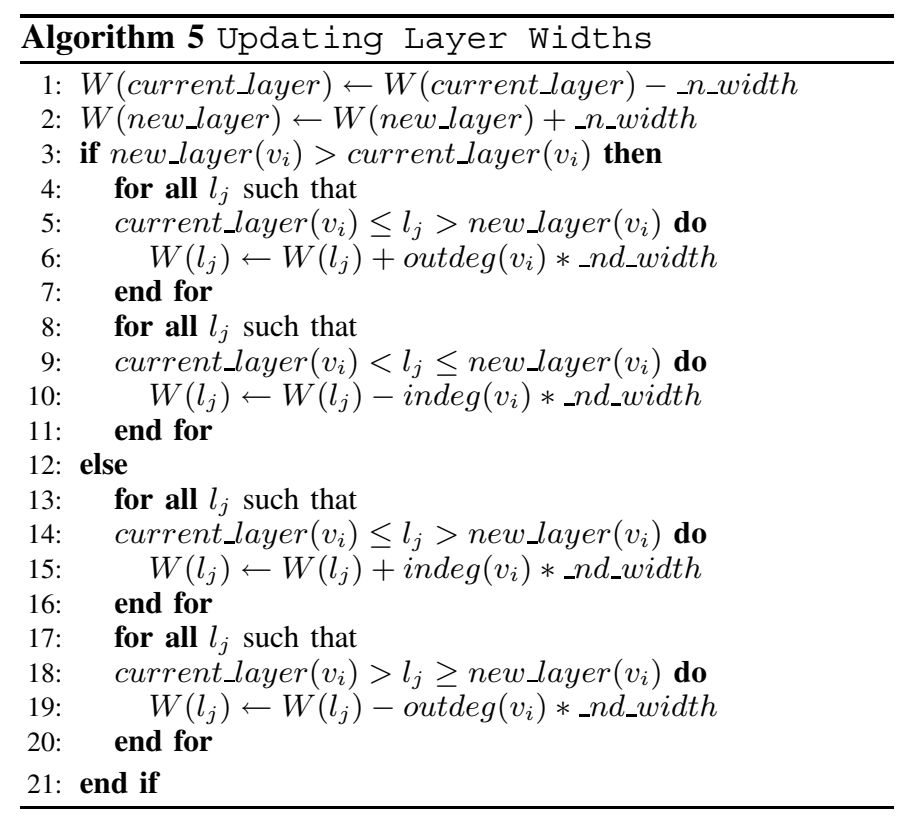

graphs. $^{2}$

First our algorithm was compared against the LPL algorithm and the MinWidth heuristic. Then the two were combined with the PL heuristic which in total resulted in four algorithms being used for the evaluation of our algorithm. The set of 1277 graphs was divided into 19 groups according to the number of vertices in each graph - ranging from 10 to 100 with step size 5. The main goal of these initial tests was to roughly evaluate the Ant Colony layering algorithm's performance and the feasibility of its further research. During the tests conducted four graph layering criteria namely - layering width, layering height, dummy vertices count (DVC), and maximum edge density plus a performance related one - algorithm's running time, were used.

The width of the Ant Colony layering compared with the other two algorithms is shown in Fig. 4 and Fig. 5. It can be seen that the width of the layerings produced by our algorithm is smaller than the the width of the LPL layerings and matches the ones resulting from the combination LPL plus PL heuristic. The layering width is even smaller when the dummy vertices contribution is not taken into account (the second plot in Fig. 4). This is a result of the fact that when an ant decides on which layer a vertex should be placed it uses as heuristic information the layer width estimation of all layer candidates by giving higher priority to the layers with fewer vertices currently. While it was somehow anticipated that our algorithm was going to produce narrower layerings than the LPL, the fact that it also matches the widths of the LPL plus the PL heuristic are rather encouraging. When compared with the MinWidth and MinWidth with PL our algorithm performs very close to these two algorithms especially in the case where the dummy vertices are taken into account (the first diagram in Fig. 5). Here the winner is MinWidth combined by PL followed

\footnotetext{
${ }^{2}$ AT\&T graphs available from http://www.graphdrawing.org
}

closely by the Ant Colony layering algorithm, which in turn shows better results than the MinWidth heuristic when run on its own. This is not the case though when the contribution of dummy vertices is not taken into account (the second diagram in Fig. 5). Here clearly the winner is MinWidth followed by the MinWidth with PL and the AntColony both showing close results.

The next criteria used were the height of the layerings and the number of dummy vertices (DVC). The results are shown in Fig. 6 and Fig. 7. The clear winner when it comes to the height of the layering is of course the LPL algorithm. The Ant Colony layerings are between 20 and 30\% higher than the LPL ones and this is a result of achieving smaller layering width than the LPL. One thing to note here is the fact that even by "stretching" the LPL layerings by those 20 to $30 \%$ our algorithm manages to keep the same number of dummy vertices as the original LPL layering (second diagram in Fig. 6). The Dummy Vertices Count (DVC) of the Ant Colony though is greater than the LPL when combined with PL.

The last two criteria used are the Edge Density (ED) and the Running Time (RT). ED is the maximum number of edges between any two layers of the resulting layering. The lower this value is the more uniform distribution of edges is observed in the final drawing of the graph we are layering. According to Fig. 8 and Fig. 9 the ED of the layerings resulting from applying the Ant Colony are between the values of the MinWidth and MinWidth with PL and are better when compared with the LPL and LPL with PL. When comparing the running times - as expected LPL and MinWidth are the winners. While this was no suprise to us it was encouraging to see that the RT of our algorithm is not much higher when LPL and MinWidth are combined with the PL heuristic.
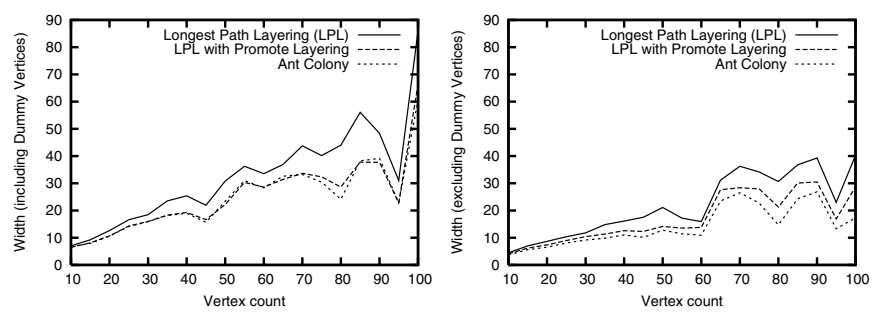

Fig. 4. Width of Ant Colony Layering Compared with LPL and LPL with PL

\section{PARAMETER TUNING}

The Ant Colony operates depending on a number of parameters that set the number of ants, tours to be performed, initial pheromone values, rate of pheromone evaporation and so on. There are two main parameters though named $\alpha$ and $\beta$ that influence the significance of the pheromone and heuristic information respectively when a decision is made by the ant. Various tests were performed for $\alpha$ and $\beta$ ranging from 1 to 5 and the best results were achieved for $\alpha=3$ and 

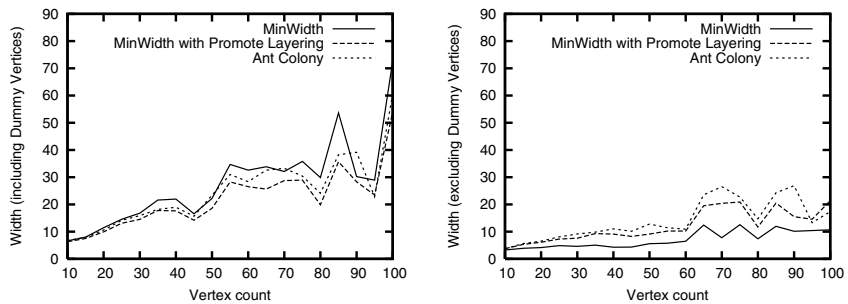

Fig. 5. Width of Ant Colony Layering Compared with MinWidth and MinWidth with PL
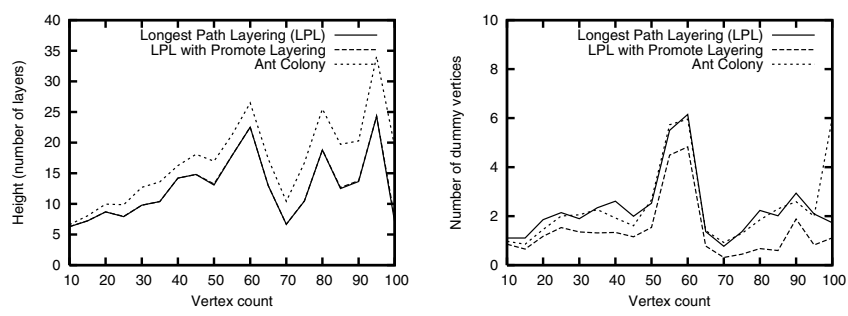

Fig. 6. Height and DVC of Ant Colony Layering Compared with LPL and LPL with PL
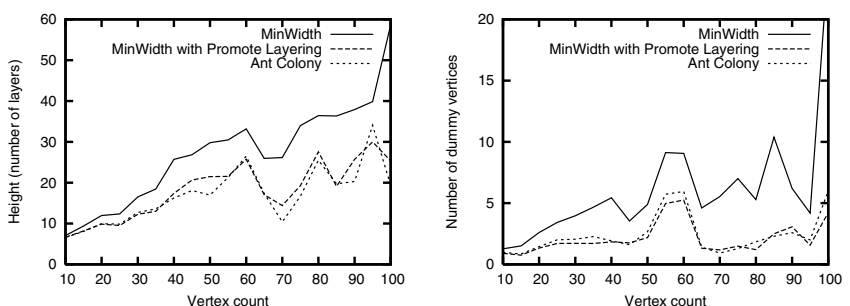

Fig. 7. Height and DVC of Ant Colony Layering Compared with MinWidth and MinWidth with PL
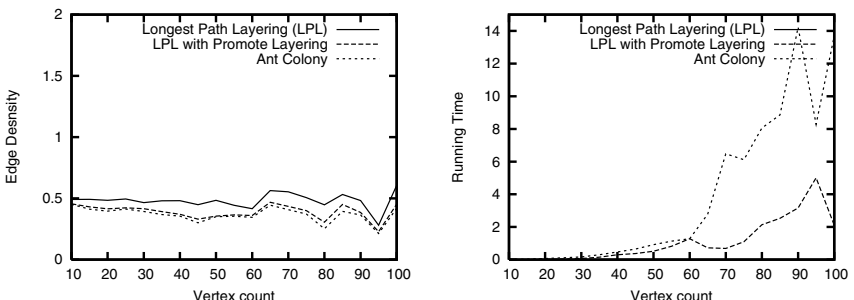

Fig. 8. Edge density and Running time of Ant Colony Layering Compared with LPL and LPL with PL
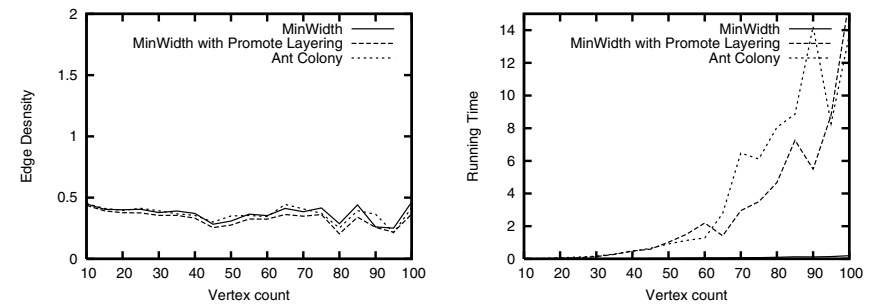

Fig. 9. Edge density and Running time of Ant Colony Layering Compared with MinWidth and MinWidth with PL $\beta=5$ followed closely by the results for $\alpha=1$ and $\beta=3$ showing slightly lower performance but at the expense of longer running times for the former. Therefore it was decided that 1 and 3 will be used as respective values for those two parameters in our further investigations. Interestingly those values for $\alpha$ and $\beta$ showed good performance of the Ant Colony Optimization heuristics when tested with instances of the Traveling Salesman Problem (TSP) as reported previously [4] Another parameter considered is the dummy vertex width (nd_width) although this is not a parameter of the Ant Colony it has, as it proved from the tests we run, a direct influence on the quality of the final layering. We run the algorithm for values for nd_width ranging from 0.1 to 1.2 with step 0.1 and the best results were achieved for _nd_width $=1.1$ closely followed by _nd_width $=1$. Again the slightly better performance for 1.1 could not justify the longer running time and therefore the _nd_width $=1$ will be used in our experiments.

\section{CONCLUSION}

On the basis of the initial tests we can conclude that Ant Colony layering algorithm performs well when compared against the two base layering methods LPL and MinWidth alone and combined with the PL heuristic. Those two layering methods target two competing layering characteristics the height (LPL) and the width (MinWidth) of a layering. The fact that the Ant Colony layering algorithm produces comparable results (only slightly worse) in the key area (height and width respectively) for each of the two algorithms and in the same time outperforms them in the other layering criteria proves that the algorithm is doing what it is supposed to do and appears to be more universal than the other two. However, the running time of the AntColony is greater than any of the two base methods. This is due to the fact that the Ant Colony layering algorithm uses LPL to build its initial layering. Nevertheless when the Ant Colony layering algorithm is compared to LPL and MinWidth with PL the running time of the first is not significantly worse than the running time of the other two algorithms.

\section{REFERENCES}

[1] G. Di Battista, P. Eades, R. Tamassia, and I. G. Tollis, Graph Drawing: Algorithms for the visualization of graphs. New Jersey, USA, Prentice Hall, Inc., 1999.

[2] E. G. Coffman and R. L. Graham, Optimal scheduling for two processor systems. Acta Informatica, Volume 1, pages 200-213, 1972.

[3] M. Dorigo, V. Maniezzo, and A. Colorni, The Ant System: Optimization by a colony of cooperating agents. IEEE Transactions on Systems, Man, and Cybernetics Part B: Cybernetics, Volume 26, pages 29-41, 1996.

[4] M. Dorigo, T. Stützle, Ant Colony Optimization. Cambridge, Massachusetts and London, England, The MIT Press 2004.

[5] E. R. Gansner and E. Koutsofios and S. C. North and Kiem-Phong Vo, A Technique for Drawing Directed Graphs. Software Engineering, Volume 19, pages 214-230, 1993.

[6] P. Heally and N. S. Nikolov, How to Layer a Directed Acyclic Graph. GD '01: Revised Papers from the 9th International Symposium on Graph Drawing, pages 16-30, Springer-Verlag, 2002.

[7] G. L. Nemhauser and L. A. Wolsey, Integer and Combinatorial Optimization. Wiley-Interscience series in discrete mathematics and optimization, John Wiley \& Sons, Inc., 1988. 
[8] N. S. Nikolov and A. Tarassov Graph layering by promotion of nodes. journal of Discrete Applied Mathematics, issue 5 IV ALIO/EURO Workshop on Applied Combinatorial Optimization, Volume 154, pages 848860, Elsevier, 2006.

[9] N. S. Nikolov, A. Tarassov, and J. Branke In Search for Efficient Heuristics for Minimum-Width Graph Layering with Consideration of Dummy Nodes. The ACM Journal on Experimental Algorithmics, Volume 10, Section: 2 - WEA'04, pages 1-27, 2005.

[10] K. Sugiyama, Graph Drawing and Applications for software and knowledge engineers. Series on Software Engineering and Knowledge Engineering, Volume 11, World Scientific, 2002.

[11] N. S. Nikolov, A Polyhedral Approach to Directed Acyclic Graph Layering. A PhD thesis, Department of Computer Science and Information Systems, University of Limerick, July 2002.

[12] K. Sugiyama, S. Tagawa, and M. Toda Methods for Visual Understanding of Hierarchical Systems. IEEE Trans. on Systems, Man, and Cybernetics, vol. SMC-11, no. 2, pp. 109-125, 1981. 